POS $\quad$ PROCEEDINGS

\title{
Domain Wall Fermion Lattice Super Yang Mills
}

\author{
Joel Giedt* \\ Department of Physics, Applied Physics and Astronomy, Rensselaer Polytechnic Institute, \\ 110 8th Street, Troy NY 12065 USA \\ E-mail: giedt jerpi.edu

\section{Richard Brower} \\ Physics Department, Boston University, 590 Commonwealth Avenue, Boston MA 02215 \\ E-mail: brower@bu.edu
}

\section{Simon Catterall}

Department of Physics, Syracuse University, Syracuse, NY 13244 USA

E-mail: smcesyracuse.edu

\section{George T. Fleming}

Department of Physics, Sloane Laboratory, Yale University, New Haven, Connecticut 06520, USA

E-mail: George.Fleming@yale.edu

\section{Pavlos Vranas}

Physical Sciences Directorate, Lawrence Livermore National Laboratory,

7000 East Ave., Livermore, CA 94550

E-mail: vranasellnl.gov

We report preliminary results of lattice super-Yang-Mills computations using domain wall fermions, performed at an actual rate of $1000 \mathrm{Gflop} / \mathrm{s}$, over the course of six months, using two BlueGene/L racks at Rensselaer's CCNI supercomputing center. This has allowed us to compute the gluino condensate and string tension over a wide range of lattice parameters, setting the stage for continuum, chiral extrapolations.

The XXVI International Symposium on Lattice Field Theory

July 14-192008

Williamsburg, Virginia, USA

\footnotetext{
*Speaker.
} 
We have used domain wall fermions (DWF) [1,2] to study nonperturbative aspects of pure $\mathscr{N}=1$ super-Yang-Mills (SYM) [3]. The strong dynamics of supersymmetric gauge theories underlie most models of spontaneous supersymmetry (SUSY) breaking, and the development of a first-principles tool is needed. Though at finite lattice spacing SUSY is violated, it is automatically recovered [4] in the continuum limit with a massless gluino. ${ }^{1}$ When the DWF formulation is employed, this "chiral limit" is achieved without the need for a computationally expensive, nonperturbatively determined fine-tuning of the bare gluino mass [5, 6]; in the limit of infinite domain wall separation $L_{s} \rightarrow \infty$, DWF realize the lattice chiral symmetry [7] associated with GinspargWilson fermions [8], which protects against additive mass renormalization. These nice features of the DWF approach are to be contrasted with the Wilson fermion formulation, which was pursued for several years by the DESY-Münster-Roma collaboration $[9,10,11,12,13,14,15]$.

Lattice studies can provide details that other approaches cannot, such as "snapshots" of the gauge field configurations that are dominating the gluino condensate. For instance, in the work of Fleming et al. [16], the only DWF simulation of SYM to date, it was suggested that spikes in the gluino condensate may correspond to configurations with fractional topological charge, as would be expected from Ref. [17]. Further pursuit of this conjecture, consisting of lattice studies of monopoles and topological charge, is on our agenda. Also, we will compute the low-lying spectrum of composite states, consisting of strongly bound gluons and gluinos. Apart from the inconclusive results of DESY-Münster-Roma, this aspect of SYM is completely unknown from continuum methods, and ideally suited to the lattice approach.

At early stages in such studies, understanding numerical behavior of important quantities such as the gluino condensate will teach us a lot about the lattice formulation that we currently do not know. For instance, it is important to set "benchmarks" regarding compute time, and lattice artifacts such as discretization and finite size effects. The first place that we will examine this is in the gluino condensate, which is believed to be known exactly by continuum methods $[18,19,20$, $17,21]$, and is therefore ideal for calibrating the lattice methods. Understanding of the lattice theory and simulation performance is already emerging from our preliminary results, as we now briefly discuss. We also mention that Endres has begun simulations of this lattice model and presented results at this meeting [22].

Domain wall fermion simulations require world-class computing resources, such as are are available to Giedt at Rensselaer; namely, the Computational Center for Nanotechnology Innovations (CCNI), one of the world's most powerful university-based supercomputing centers, and a top 25 supercomputing center of any kind in the world. We are presently the third heaviest user of this facility, and have been generating lattice configurations and measurements continuously at a sustained actual rate of $1000 \mathrm{Gflop} / \mathrm{s}$ since the end of January 2008. For comparison the DESYMünster-Roma collaboration performed their computations at a rate of $10 \mathrm{Gflop} / \mathrm{s}$ for a cumulative time of one year. Thus our study represents a hundred-fold improvement over what has been done

\footnotetext{
${ }^{1}$ Here we use the terms "gluon" and "gluino" by way of analogy. It should be kept in mind that the strongly coupled gauge theory would be an extension to the gauge group of the Standard Model.
} 


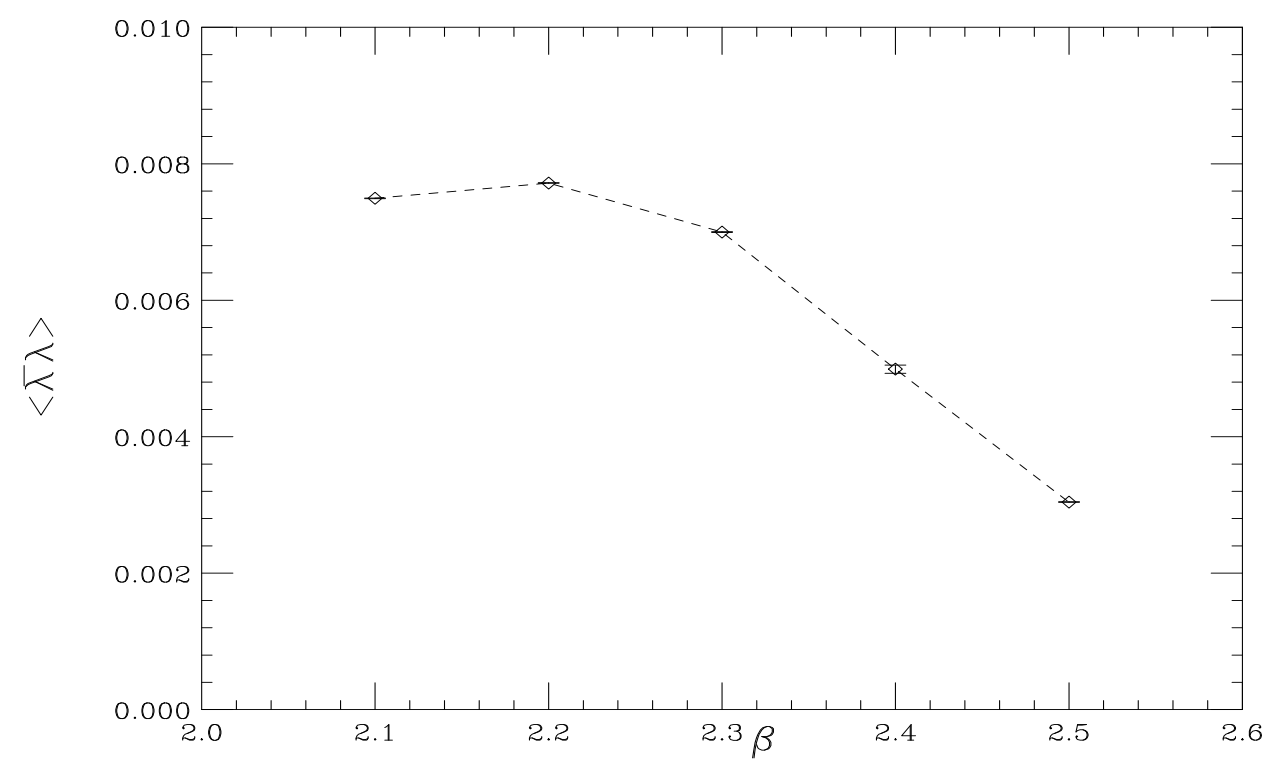

Figure 1: The gluino condensate versus $\beta$ for a $16^{3} \times 32$ lattice with domain wall separation $L_{s}=16$ (dashed line drawn to guide the eye). It can be estimated from the figure that for the $16^{3} \times 32$ lattice studied here, the system will deconfine at $\beta \sim 2.7$, as a result of finite size effects.

previously, just in terms of raw computation power.

As an example of our results, we have obtained the bare gluino condensate from dynamical domain wall fermion simulations for a variety of bare gauge couplings $g$, parameterized in terms of $\beta=4 / g^{2}$, as is conventional in $\mathrm{SU}(2)$ lattice gauge theory. The results for a $16^{3} \times 32$ lattice (i.e., the number of sites in spatial and temporal directions) with domain wall separation $L_{s}=16$ sites are displayed in Fig. 1. We note that such data for the condensate versus $\beta$ has never been obtained before; it is important because the continuum limit corresponds to $\beta \rightarrow \infty$ (with the physical size of the lattice held fixed). A fit to the data obviously yields a vanishing condensate at a finite gauge coupling $\beta \sim 2$.7. This just reflects the fact that as $\beta$ increases the lattice spacing shrinks, and thus so does the physical size of the lattice in its entirety. In a small enough "box" confinement will disappear and the condensate "melts." Thus we already gain an important benchmark: to go much beyond $\beta=2.5$ will require larger lattices, and in fact one should carefully measure systematic errors due to finite size effects at $\beta \approx 2.5$. This is consistent with what is already known from the so-called "quenched" theory, which has no gluinos.

Fig. 2 shows the gluino condensate for decreasing values of the residual mass $m_{\text {res }}$, which is a measure of explicit chiral symmetry breaking due to finite $L_{s}$ [23]. As expected, larger $L_{s}$ values have the smallest $m_{\text {res }}$, and a nonzero gluino condensate appears to occur in the $m_{\text {res }} \rightarrow \infty$ limit. Also as expected, smaller values of $m_{\text {res }}$ occur for the weaker coupling $\beta=2.4$.

Finally, we have looked at Creutz ratios [24],

$$
\chi(R, R)=-\ln \frac{W(R, R) W(R-1, R-1)}{W(R, R-1)^{2}} \sim \sigma a^{2},
$$




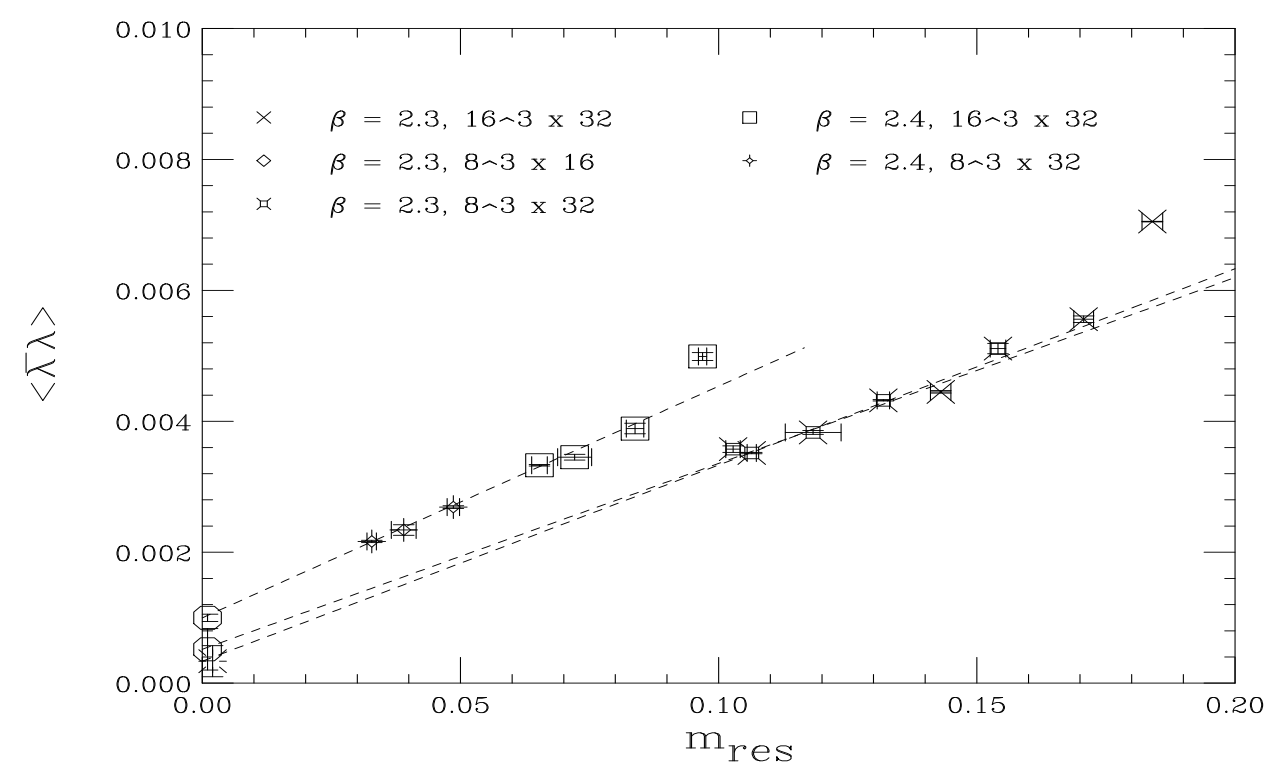

Figure 2: Our simulation results for the gluino condensate versus $m_{\text {res }}$, where the latter is a measure of explicit chiral symmetry breaking due to finite domain wall separation $L_{S}$. Linear extrapolations are also shown.

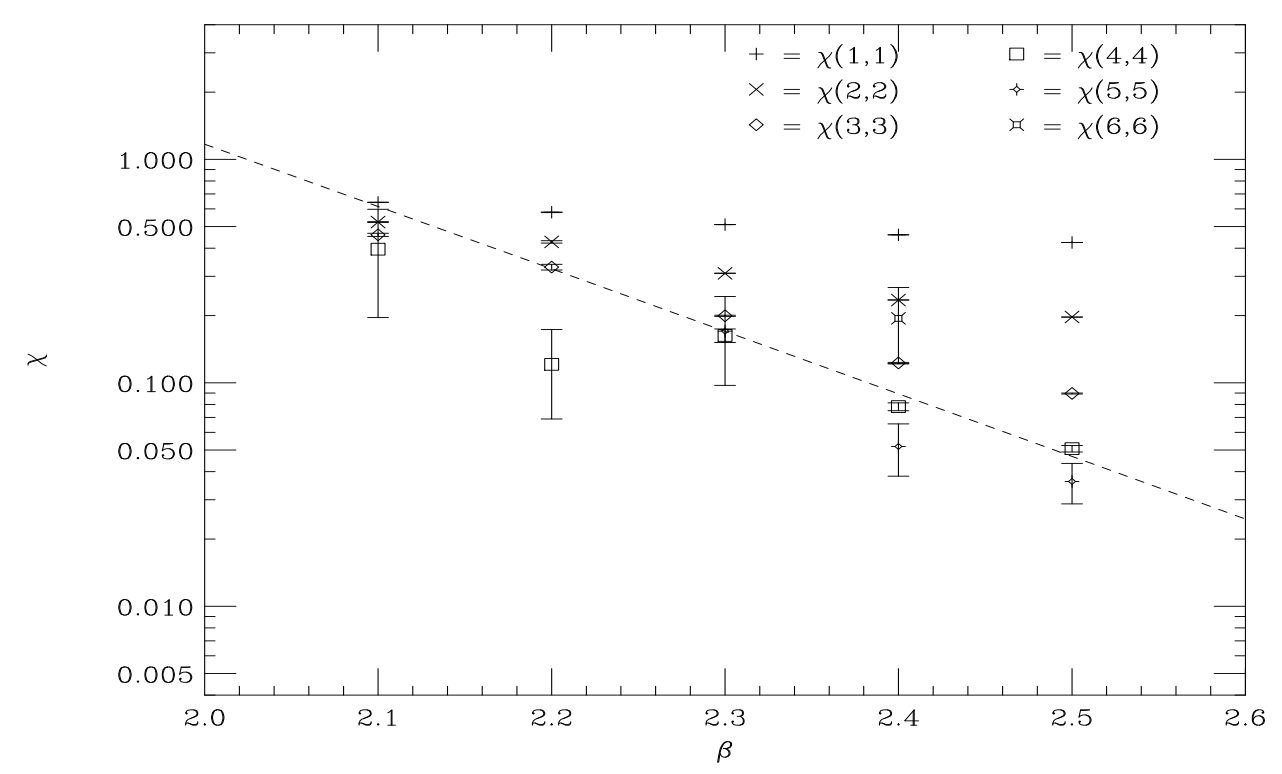

Figure 3: Creutz ratios for the $16^{3} \times 32$ lattice with $L_{s}=16$. The dashed line indicates the 2-loop SUSY prediction for the dependence $\chi \sim \sigma a^{2}$ where $\sigma$ is the string tension and $a=a(\beta)$ is the lattice spacing. 
where $W\left(R, R^{\prime}\right)$ is an $R \times R^{\prime}$ Wilson loop, in order to extract the string tension $\sigma$ in lattice units, as well as to delineate the scaling regime where the continuum limit may be extracted. In the process we obtain an estimate of the lattice spacing in units of the string tension. Results for the $16^{3} \times 32$, $L_{s}=16$ lattice are shown in Fig. 3. Although the errors are somewhat large, scaling is clearly setting in at around $\beta=2.3$. To see this one notes that the larger Creutz ratios appear to coalesce on an envelope, corresponding to the distance scale at which an area law begins to take hold in the Wilson loops.

We emphasize that all of the results mentioned above are ground-breaking as far as lattice SYM is concerned. Several important goals related to the lattice SYM project have already been achieved, laying the groundwork for the more extensive studies that will follow:

- Developed parallel simulation code for SYM by modification of the current version of the Columbia Physics System (CPS) QCD package.

- This extends DOE funded code (CPS, part of USQCD's SciDAC program) to "beyond the Standard Model" physics, which is a realization of one of the USQCD Collaboration objectives [25].

- We have reproduced the results of [16] as a check on our code.

- Our software runs successfully on IBM's Bluegene (BG) architecture, taking full advantage of BG specific communications utilities.

- Developed Landau gauge-fixing and Fourier space propagator code for adjoint fermion representations, essential for nonperturbative renormalization of the condensate, in the RI/MOM scheme $[26,27]$.

- Established timing and statistical uncertainty benchmarks. For example, we have found that for small single-node volumes $\left(16^{3} \times 32 \times L_{s} / 2048 \mathrm{CPU}\right.$ 's $=64 \times L_{s}$ sites per CPU $)$ the efficiency of the BG parallel code is 10 percent.

In the course of our studies, we intend to investigate other domain wall fermion formulations (e.g., "gap" [28] and "Mobius" [29]) as ways to approach the chiral limit more quickly. Also, we will implement recent optimizations of fermion matrix inverters (to increase efficiency) and improved actions (to reduce systematic errors).

\section{Acknowledgements}

We benefited from a copy of the code that was used in [16], and employed it as the basis for our modifications to the current version of the Columbia Physics System. At various points JG benefited from technical assistance provided by Chulwoo Jung (Brookhaven National Lab) and Adam Todorski (SCOREC and CCNI at Rensselaer). The computational efforts on this project mainly utilized the Computational Center for Nanotechnology Innovations (CCNI), and JG expresses his 
appreciation for continuous access to that facility. The project also, at times, utilized the SUR BlueGene/L at Rensselaer, which is supported by NSF grant 0420703 entitled "MRI: Acquisition of Infrastructure for Research in Grid Computing and Multiscale Systems Computation" and a gift by the IBM Corporation of a BlueGene/L computer. JG acknowledges support from Rensselaer faculty development funds.

\section{References}

[1] D. B. Kaplan, "A Method for simulating chiral fermions on the lattice," Phys. Lett. B 288 (1992) 342 [arXiv:hep-lat/9206013].

[2] Y. Shamir, "Chiral fermions from lattice boundaries," Nucl. Phys. B 406 (1993) 90 [arXiv:hep-lat/9303005].

[3] S. Ferrara and B. Zumino, “Supergauge Invariant Yang-Mills Theories,” Nucl. Phys. B 79 (1974) 413.

[4] G. Curci and G. Veneziano, "Supersymmetry And The Lattice: A Reconciliation?," Nucl. Phys. B 292 (1987) 555.

[5] H. Neuberger, "Vector like gauge theories with almost massless fermions on the lattice," Phys. Rev. D 57 (1998) 5417 [arXiv:hep-lat/9710089].

[6] D. B. Kaplan and M. Schmaltz, "Supersymmetric Yang-Mills theories from domain wall fermions," Chin. J. Phys. 38 (2000) 543 [arXiv:hep-lat/0002030].

[7] M. Luscher, "Exact chiral symmetry on the lattice and the Ginsparg-Wilson relation," Phys. Lett. B 428 (1998) 342 [arXiv:hep-lat/9802011].

[8] P. H. Ginsparg and K. G. Wilson, "A Remnant Of Chiral Symmetry On The Lattice," Phys. Rev. D 25 (1982) 2649.

[9] I. Campos, R. Kirchner, I. Montvay, J. Westphalen, A. Feo, S. Luckmann, G. Münster, K. Spanderan [DESY-Munster Collaboration], "Monte Carlo simulation of SU(2) Yang-Mills theory with light gluinos,” Eur. Phys. J. C 11 (1999) 507 [arXiv:hep-lat/9903014].

[10] F. Farchioni, C. Gebert, R. Kirchner, I. Montvay, A. Feo, G. Münster, T. Galla, A. Vladikas [DESY-Munster-Roma Collaboration], “The supersymmetric Ward identities on the lattice," Eur. Phys. J. C 23 (2002) 719 [arXiv:hep-lat/0111008].

[11] I. Montvay, "Supersymmetric Yang-Mills theory on the lattice," Int. J. Mod. Phys. A 17 (2002) 2377 [arXiv:hep-lat/0112007].

[12] R. Peetz, F. Farchioni, C. Gebert and G. Munster, "Spectrum of SU(2) SUSY Yang-Mills theory with a light gluino," Nucl. Phys. Proc. Suppl. 119 (2003) 912 [arXiv:hep-lat/0209065].

[13] F. Farchioni, G. Muenster and R. Peetz, "The volume source technique for flavor singlets: A second look,” Eur. Phys. J. C 38 (2004) 329 [arXiv:hep-lat/0404004].

[14] F. Farchioni and R. Peetz, "The low-lying mass spectrum of the N = 1 SU(2) SUSY Yang-Mills theory with Wilson fermions," Eur. Phys. J. C 39 (2005) 87 [arXiv:hep-lat/0407036]. 
[15] R. Peetz, "Spectrum of $\mathscr{N}=1$ Super Yang Mills Theory on the Lattice with a Light Gluino," doctoral dissertation, University of Münster, Germany, 2003, available at: http://deposit.ddb.de/cgi-bin/dokserv?idn=97018249X.

[16] G. T. Fleming, J. B. Kogut and P. M. Vranas, "Super Yang-Mills on the lattice with domain wall fermions," Phys. Rev. D 64 (2001) 034510 [arXiv:hep-lat/0008009].

[17] N. M. Davies, T. J. Hollowood, V. V. Khoze and M. P. Mattis, "Gluino condensate and magnetic monopoles in supersymmetric gluodynamics,” Nucl. Phys. B 559 (1999) 123 [arXiv:hep-th/9905015].

[18] I. Affleck, M. Dine and N. Seiberg, "Dynamical Supersymmetry Breaking In Supersymmetric QCD," Nucl. Phys. B 241 (1984) 493.

[19] I. Affleck, M. Dine and N. Seiberg, "Dynamical Supersymmetry Breaking In Four-Dimensions And Its Phenomenological Implications,” Nucl. Phys. B 256 (1985) 557.

[20] V. A. Novikov, M. A. Shifman, A. I. Vainshtein and V. I. Zakharov, "Supersymmetric instanton calculus: Gauge theories with matter,” Nucl. Phys. B 260 (1985) 157 [Yad. Fiz. 42 (1985) 1499].

[21] F. Cachazo, M. R. Douglas, N. Seiberg and E. Witten, "Chiral rings and anomalies in supersymmetric gauge theory," JHEP 0212 (2002) 071 [arXiv:hep-th/0211170].

[22] M. Endres, "Numerical simulation of $\mathscr{N}=1$ supersymmetric Yang-Mills theory," to appear in proceedings "The XXVI International Symposium of Lattice Field Theory," College of William and Mary, Williamsburg, VA, July 14-19, 2008.

[23] T. Blum et al., "Quenched lattice QCD with domain wall fermions and the chiral limit," Phys. Rev. D 69 (2004) 074502 [arXiv:hep-lat/0007038].

[24] M. Creutz, “Asymptotic Freedom Scales,” Phys. Rev. Lett. 45 (1980) 313.

[25] R. Brower, N. Christ, M. Creutz, P. Mackenzie, J. Negele, C. Rebbi, D. Richards, S. Sharpe, R. Sugar [Lattice QCD Executive Committee, USQCD Collaboration], "Lattice Gauge Theory for Physics Beyond the Standard Model," whitepaper available at: http: / / www. usqcd.org/documents/bsm.pdf.

[26] G. Martinelli, C. Pittori, C. T. Sachrajda, M. Testa and A. Vladikas, "A General Method For Nonperturbative Renormalization Of Lattice Operators,” Nucl. Phys. B 445 (1995) 81 [arXiv:hep-lat/9411010].

[27] T. Blum et al., "Non-perturbative renormalisation of domain wall fermions: Quark bilinears," Phys. Rev. D 66 (2002) 014504 [arXiv:hep-lat/0102005].

[28] P. M. Vranas, “Gap domain wall fermions,” Phys. Rev. D 74 (2006) 034512 [arXiv:hep-lat/0606014].

[29] R. C. Brower, H. Neff and K. Orginos, "Moebius fermions: Improved domain wall chiral fermions," Nucl. Phys. Proc. Suppl. 140 (2005) 686 [arXiv:hep-lat/0409118]. 